\title{
Bose-Einstein effects in multiplicity and net-charge correlations in pp collisions using Pythia 8 simulations
}

\author{
Dmitry Neverov* ${ }^{\dagger}$ \\ Saint-Petersburg State University \\ E-mail: D.I.Neverov@gmail.com
}

\begin{abstract}
Correlations between various observables, e.g. multiplicities of particles produced in pp collisions at the LHC energies within intervals separated in pseudo-rapidity and azimuth angle, could be a sensitive tool to analyze hadron collisions dynamics and test hadron production models.

In this report we present results of studies of multiplicity correlation coefficient topology for like- and unlike-sign pairs of charged particles using PYTHIA8 event generator. The correlation coefficients were extracted using long-range forward-backward correlation method.

Peculiar behavior of correlation coefficient topology of net-charge is obtained in short-range region. Similar peculiarities are predicted in balance functions [1]. Analysis shows that effects of Bose-Einstein statistics have a strong influence in this region of such correlations.

The results indicate the necessity of experimental studies of net-charge correlation topology that could bring new constraints to PYTHIA8 tunes.
\end{abstract}

XXII International Baldin Seminar on High Energy Physics Problems

15-20 September, 2014

JINR, Dubna, Russia

\footnotetext{
* Speaker.

${ }^{\dagger}$ The work was supported by St.Petersburg State University grant 11.38.197.2014.
} 


\section{The method}

In the current analysis forward-backward (F-B) correlation method [2] was used to measure net-charge angular correlations. In this method two small windows of widths $\delta \eta$ in pseudo-rapidity and $\delta \phi$ in azimuth angle are picked with their centers at $\left(\eta_{1}, \phi_{1}\right)$ and $\left(\eta_{2}, \phi_{2}\right)$, named backward (B) and forward (F) respectively. Desired observables, e.g. $A$ in $F$ window and $B$ in $B$ window, are calculated for each window combination in each event. Correlation function is then obtained as

$$
b\left(\eta_{1}, \phi_{1}, \eta_{2}, \phi_{2}\right)=\frac{\langle A B\rangle-\langle A\rangle\langle B\rangle}{\left\langle A^{2}\right\rangle-\langle A\rangle^{2}}
$$

If translational invariance in $\eta$ takes place, i.e. $A$ and $B$ distributions over $\eta$ are flat, and with the rotational invariance in $\phi$ correlation function only depends on the distance between windows

$$
b\left(\eta_{1}, \phi_{1}, \eta_{2}, \phi_{2}\right)=b\left(\eta_{1}-\eta_{2}, \phi_{1}-\phi_{2}\right)=b\left(\eta_{\text {sep }}, \phi_{\text {sep }}\right)
$$

It can be shown [3] that for the case of $A$ and $B$ being particle multiplicities $N_{F}$ and $N_{B}$ this way of defining correlation function is connected to usual $C_{2}$ function used in theoretical papers [4] and that for small windows

$$
b\left(\eta_{\text {sep }}, \phi_{\text {sep }}\right) \approx\left\langle N_{B}\right\rangle C_{2}\left(\eta_{\text {sep }}, \phi_{\text {sep }}\right)
$$

Net-charge is simply a difference between the number of positively charged particles $N_{+}$and the number of negative ones $N_{-}$

$$
Q=N_{+}-N_{-}
$$

Thus to obtain net-charge correlations we have taken $Q_{F}$ as $A$ and $Q_{B}$ as $B$ in this method.

\section{Net-charge and balance functions}

The data analyzed in the current paper is $3 \mathrm{mln}$. events of pp collisions at $7 \mathrm{TeV}$ generated with PYTHIA 8.186 [5] with the Monash 2013 tune and Bose-Einstein effects turned on. BE effects are implemented in pythia as pulling identical particles' momenta closer to each other and all the momenta away afterwards to satisfy energy and momentum conservation [6].

Window's widths have been chosen $\delta \eta=0.2, \delta \phi=\pi / 4$

Particles falling into ALICE acceptance were considered $-1.6<\eta<1.6$, with $0.3 \mathrm{GeV} / \mathrm{c}<P_{T}<$ $3.0 \mathrm{GeV} / \mathrm{c}$ and no detector efficiency was simulated.

Results are presented in Fig. 1.

Note that net-charge correlation function was cut in the the short range region due to autocorrelations that go to unity and appear when forward and backward windows overlap. Nevertheless there is a clear bump around this cut points, which correspond to a dip in the balance function.

\section{Multiplicity correlations}

Because effects of Bose-Einstein statistics pull identical particle's momenta closer to each other correlations of like-signed particle multiplicities, i.e. $N_{+}$to $N_{+}$or $N_{-}$to $N_{-}$, are steeper than unlike-signed ones (Fig. 2) since latter doesn't have identical particles included. 

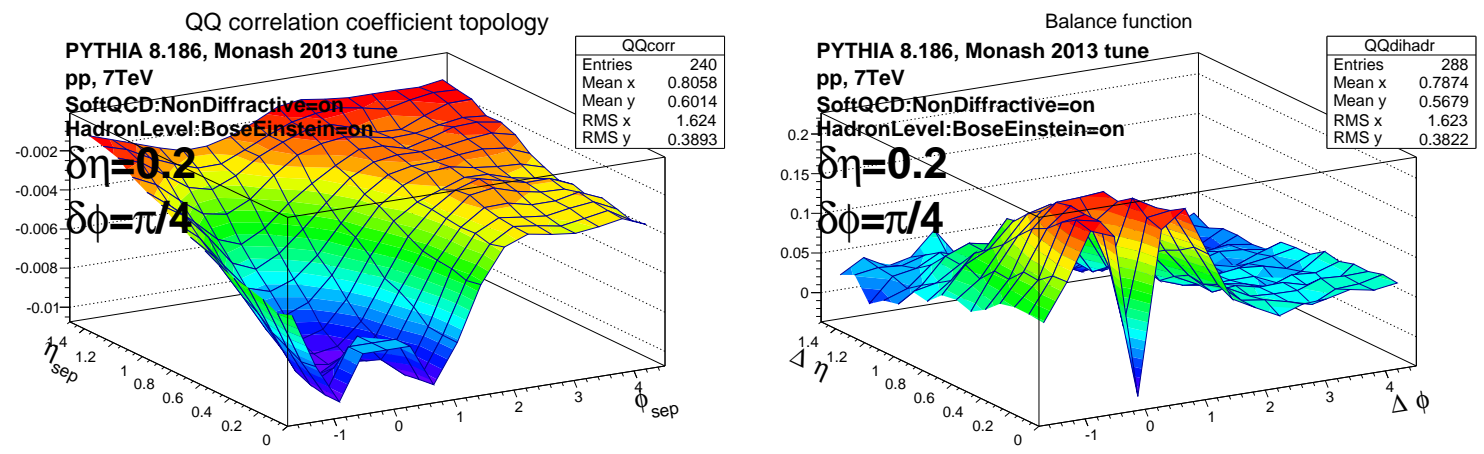

Figure 1: Net-charge correlation and balance function, $0.3 \mathrm{GeV} / \mathrm{c}<P_{T}<3.0 \mathrm{GeV} / \mathrm{c}$, BE:on
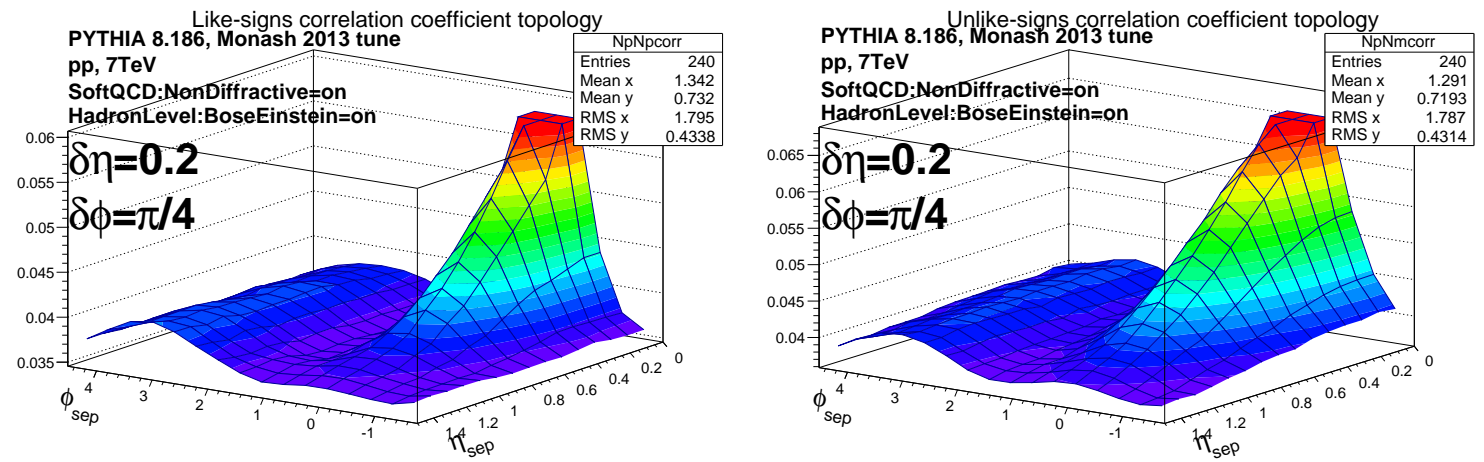

Figure 2: Like-signed an unlike-signed multiplicity correlations, $0.3 \mathrm{GeV} / \mathrm{c}<P_{T}<3.0 \mathrm{GeV} / \mathrm{c}$, BE:on

It is visible from Fig. 2 that $b_{L S}\left(\eta_{s e p}, \phi_{s e p}\right)$ has a different behavior in the short range region compared to $b_{U S}\left(\eta_{\text {sep }}, \phi_{s e p}\right)$. This explains the origin of the bump in the net-charge correlation function, since

$$
b_{Q Q}=\frac{\left\langle Q_{F} Q_{B}\right\rangle-\left\langle Q_{F}\right\rangle\left\langle Q_{B}\right\rangle}{\left\langle Q_{F}^{2}\right\rangle-\left\langle Q_{F}\right\rangle^{2}}=\frac{\left\langle\left(N_{+F}-N_{-F}\right)\left(N_{+B}-N_{-B}\right)\right\rangle-\left\langle N_{+F}-N_{-F}\right\rangle\left\langle N_{+B}-N_{-B}\right\rangle}{\left\langle\left(N_{+F}-N_{-F}\right)^{2}\right\rangle-\left\langle\left(N_{+F}-N_{-F}\right)\right\rangle^{2}}
$$

Assuming charge symmetry we get

$$
b_{Q Q}=\frac{2 \operatorname{Cov}\left(N_{+F}, N_{+B}\right)-2 \operatorname{Cov}\left(N_{+F}, N_{-B}\right)}{2 D\left(N_{+F}\right)-2 \operatorname{Cov}\left(N_{+F}, N_{-F}\right)}=\left(b_{L S}^{F-B}-b_{U S}^{F-B}\right) \frac{1}{1-b_{U S}^{F-F}} \approx b_{L S}-b_{U S}
$$

Where $\operatorname{Cov}(A, B)=\langle A B\rangle-\langle A\rangle\langle B\rangle$ and $D(A)=\left\langle A^{2}\right\rangle-\langle A\rangle^{2}$

Thus the bump in net-charge correlation function corresponds to different behavior of $b_{L S}$ and $b_{U S}$ in the short range region.

\section{Modifying physics}

To check that it is Bose-Einstein effects that solely provide the bump in net-charge correlations or the dip in balance functions another set of data was analyzed, which was generated with all the same parameters but Bose-Einstein effects were turned off. Fig. 3 and Fig. 4 

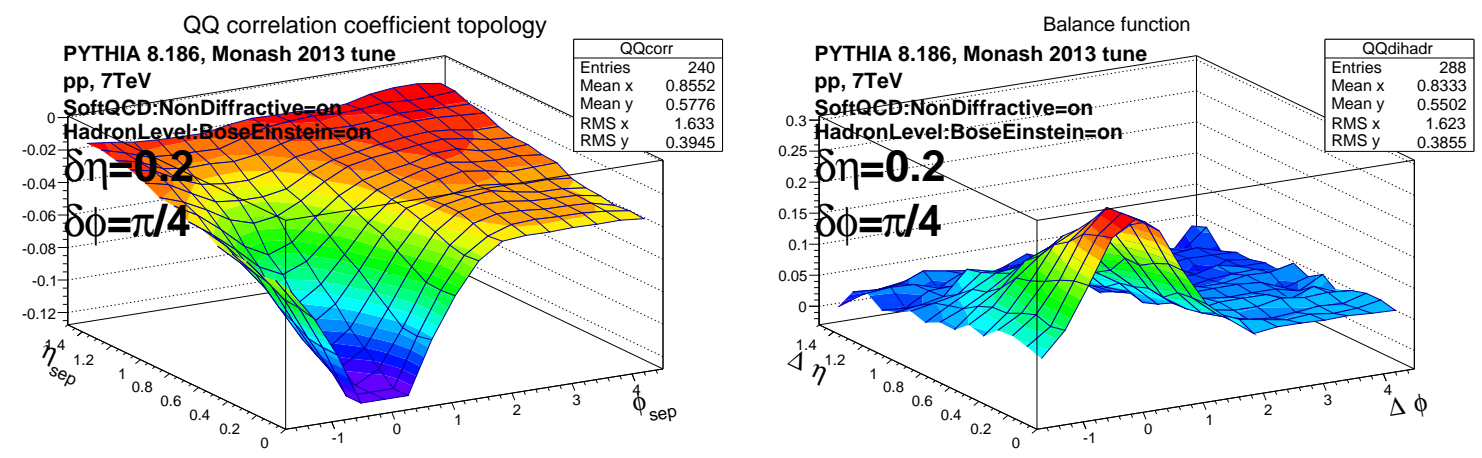

Figure 3: Net-charge correlation and balance function, $0.3 \mathrm{GeV} / c<P_{T}<3.0 \mathrm{GeV} / c$, BE:off
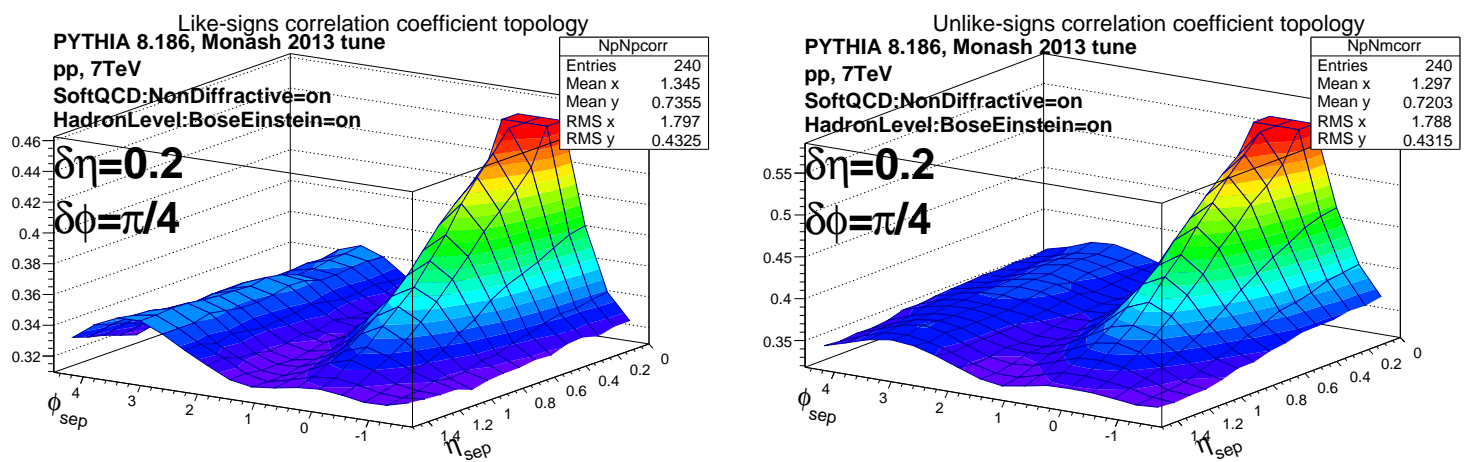

Figure 4: Like-signed an unlike-signed multiplicity correlations, $0.3 \mathrm{GeV} / c<P_{T}<3.0 \mathrm{GeV} / c$, BE:off

Comparing this figures with Fig. 1 and Fig. 2 it is clear that without Bose-Einstein $b_{L S}$ and $b_{U S}$ have similar topology and the bump disappears.

\section{Different $P_{T}$ regions}

Another thing that have been done in the current work is to check that BE effects influence on correlation functions are significant only at low $P_{T}$. Three different $P_{T}$ regions were taken and it turned out that $\mathrm{BE}$ effects influence on net-charge correlations almost vanishes if one consider particles with $P_{T}>1 \mathrm{GeV} / \mathrm{c}$ Fig. 5, 6 and 7

\section{Conclusions}

It is clear that the effects of Bose-Einstein statistics dominate like-signed particles multiplicity correlation function at low $P_{T}$ in the short range region and have no influence on unlike-signed ones. Therefore net-charge correlation within whole $P_{T}$ range has a visible bump corresponding to a similar dip in the short range region of balance function.

\section{References}

[1] Sangyong Jeon, Scott Pratt, Phys.Rev. C65 (2002) 044902 

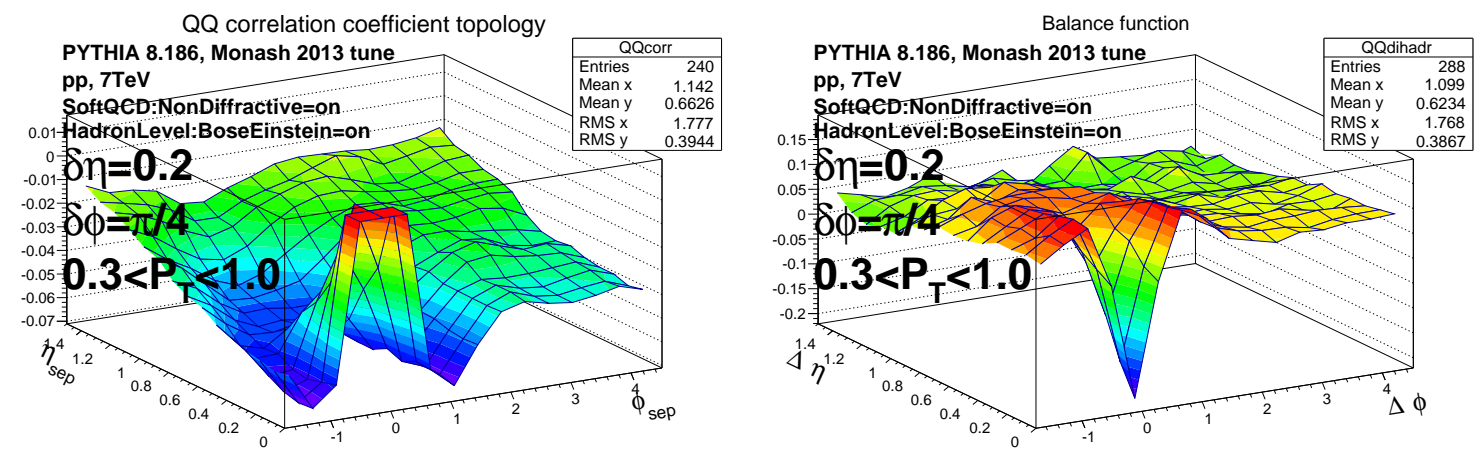

Figure 5: Net-charge correlation and balance function, $0.3 \mathrm{GeV} / \mathrm{c}<P_{T}<1.0 \mathrm{GeV} / \mathrm{c}$
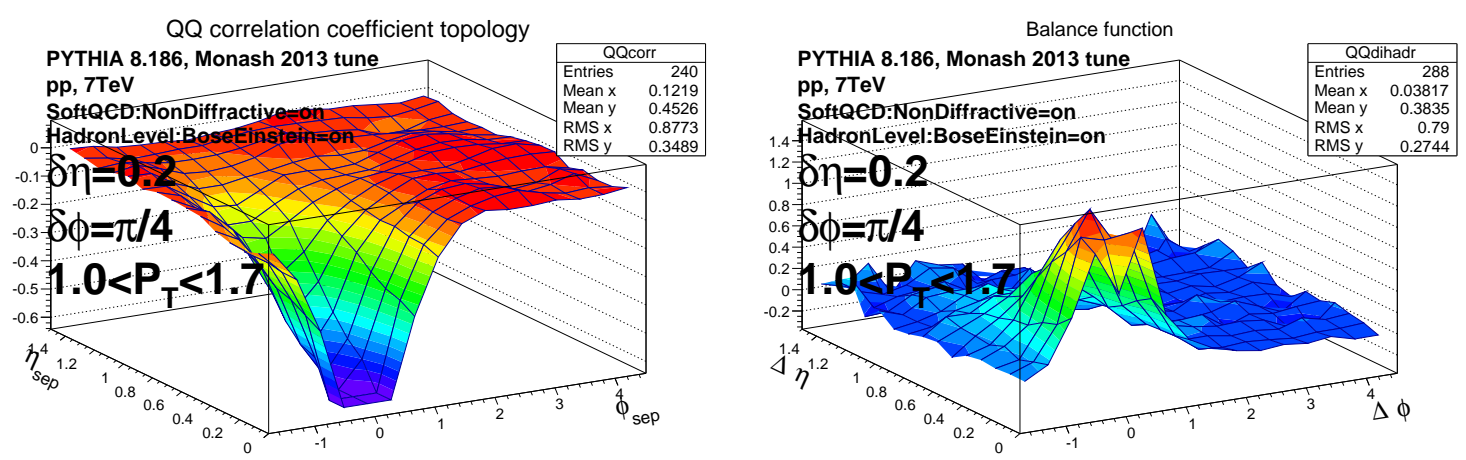

Figure 6: Net-charge correlation and balance function, $1.0 \mathrm{GeV} / \mathrm{c}<P_{T}<1.7 \mathrm{GeV} / \mathrm{c}$
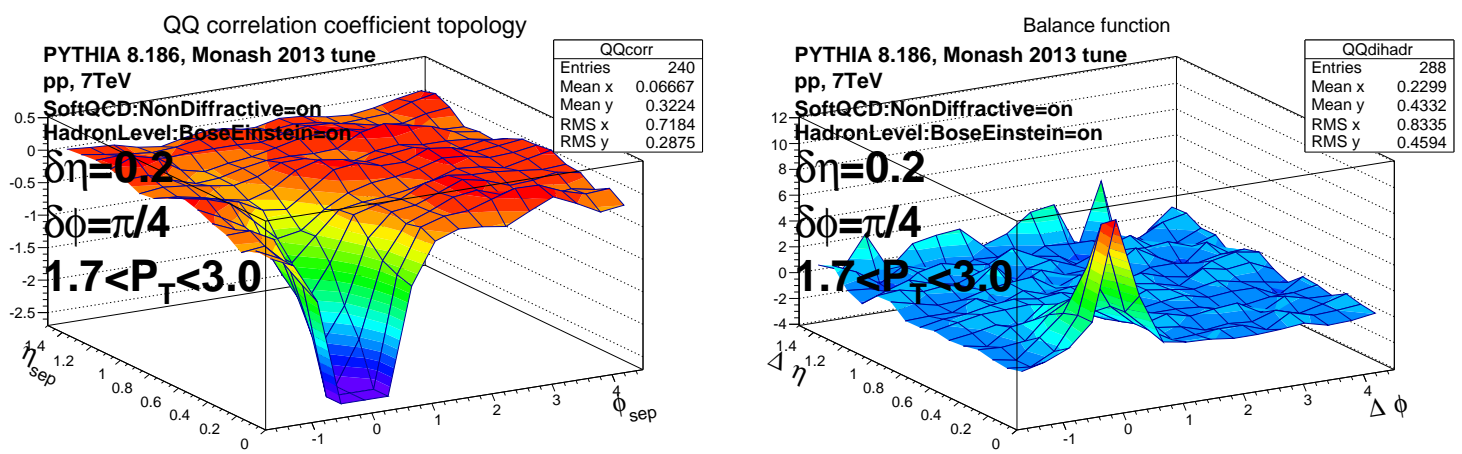

Figure 7: Net-charge correlation and balance function, $1.7 \mathrm{GeV} / \mathrm{c}<P_{T}<3.0 \mathrm{GeV} / \mathrm{c}$

[2] ALICE collaboration, ALICE Physics Performance Report, vol. 2, part 2 (6.5.15, Long-range correlations, pp. 452-455), CERN/LHCC 2005-030, 2005

[3] V. Vechernin, On description of the correlation between multiplicities in windows separated in azimuth and rapidity, PoS(QFTHEP 2013)055

[4] A. Capella, A. Krzywicki, Phys. Rev. D 18 (1978) 4120

[5] Pythia homepage

[6] L. Lonnblad, T. Sjostrand, Phys. Lett. B351 (1995) 293, Eur. Phys. J. C2 (1998) 165 